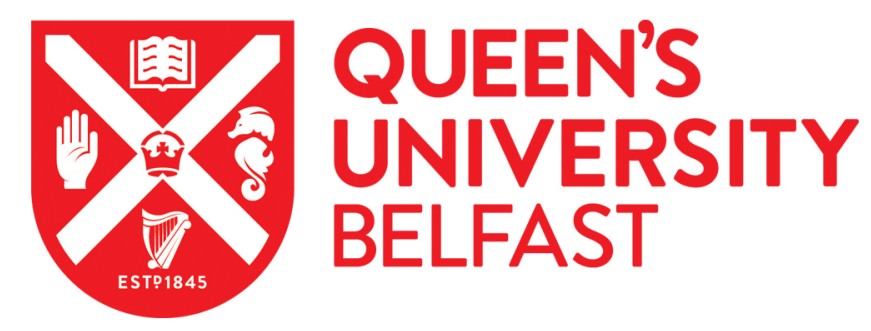

\title{
Multi-Attribute SCADA-Specific Intrusion Detection System for Power Networks
}

\author{
Yang, Y., McLaughlin, K., Sezer, S., Littler, T., Im, E. G., Pranggono, B., \& Wang, H. F. (2014). Multi-Attribute \\ SCADA-Specific Intrusion Detection System for Power Networks. leee Transactions On Power Delivery, 29(3), \\ 1092-1102. https://doi.org/10.1109/TPWRD.2014.2300099
}

\section{Published in:}

leee Transactions On Power Delivery

\section{Document Version:}

Peer reviewed version

Queen's University Belfast - Research Portal:

Link to publication record in Queen's University Belfast Research Portal

\begin{abstract}
Publisher rights
( $) 2014$ IEEE. Personal use of this material is permitted. Permission from IEEE must be obtained for all other uses, in any current or future media, including

reprinting/republishing this material for advertising or promotional purposes, creating new collective works, for resale or redistribution to servers or lists, or reuse of any copyrighted component of this work in other works.
\end{abstract}

\section{General rights}

Copyright for the publications made accessible via the Queen's University Belfast Research Portal is retained by the author(s) and / or other copyright owners and it is a condition of accessing these publications that users recognise and abide by the legal requirements associated with these rights.

Take down policy

The Research Portal is Queen's institutional repository that provides access to Queen's research output. Every effort has been made to ensure that content in the Research Portal does not infringe any person's rights, or applicable UK laws. If you discover content in the Research Portal that you believe breaches copyright or violates any law, please contact openaccess@qub.ac.uk. 


\title{
Multi-Attribute SCADA-Specific Intrusion Detection System for Power Networks
}

\author{
Y. Yang, K. McLaughlin, S. Sezer, T. Littler, E. G. Im, B. Pranggono, and H. F. Wang
}

\begin{abstract}
The increased interconnectivity and complexity of Supervisory Control and Data Acquisition (SCADA) systems in power system networks has exposed the systems to a multitude of potential vulnerabilities. In this paper we present a novel approach for a next generation SCADA-specific Intrusion Detection System (IDS). The proposed system analyses multiple attributes in order to provide a comprehensive solution able to mitigate varied cyber attack threats. The multi-attribute IDS comprises a heterogeneous whitelist and behaviour-based concept in order to make SCADA cyber systems more secure. This paper also proposes a multilayer cyber-security framework based on IDS for protecting SCADA cyber-security in Smart Grids without compromising the availability of normal data. In addition, this paper presents a SCADA-specific cyber-security test-bed to investigate simulated attacks and which has been used in the paper to validate the proposed approach.
\end{abstract}

Index Terms-- Smart Grid, SCADA, Cyber-security, Intrusion Detection

\section{INTRODUCTION}

Supervisory Control and Data Acquisition (SCADA) systems have long played a significant role in power system operation, becoming increasingly complex and interconnected as state-of-the-art information and communication technologies (ICT) are adopted. The increased complexity and interconnection of SCADA systems have exposed them to a wide range of cyber-security vulnerabilities. Furthermore, SCADA systems with legacy devices lack inbuilt cybersecurity consideration, which has resulted in serious cybersecurity vulnerable points. In practice, unauthorised or malicious access from outside sources, using IP-driven proprietary or local area networks can threaten SCADA systems by exploiting communication weaknesses to launch simple or elaborate attacks which may lead to denial of service, deliberate maloperation or catastrophic failure, and consequently compromise the safety and stability of power system operations. Thus the requirement to strengthen cybersecurity in SCADA as part of smarter grids, in particular, is a pertinent priority to ensure reliable operation and govern system stability in terms of communications integrity.

In recent years, malicious cyber-security incidents have occurred in SCADA systems. For instance, in July 2010, the Stuxnet worm attacked the Siemens SIMATIC WinCC SCADA system and physical Programmable Logic Controllers
(PLCs), exploiting a number of vulnerabilities including at least four in the Microsoft Windows operating system. It is the most famous malware attack to have damaged an industrial infrastructure directly. According to Symantec's statistics, approximately 45,000 systems around the world have been infected by the worm including Iranian nuclear facilities [1]. Many utilities remain concerned at the possibility of "collateral damage" to their infrastructures from Stuxnet-like attacks in the future.

In the early history of SCADA systems it was widely believed that such systems were secure in cyber space since they were air-gapped - that is, physically isolated from public networks. In other words, only physical security was a concern rather than cyber-security. Stuxnet crossed both the cyber and physical world by manipulating the control system of the critical infrastructure, demonstrating that "security by obscurity" is no longer a valid approach.

With the application of IT technologies, new cyber vulnerabilities will emerge in Smart Grids and similar critical infrastructures. These vulnerabilities could be exploited, not only from outside sources, such as terrorists, hackers, competitors or industrial espionage, but also from inside threats, such as ex-employees, disgruntled employees, third part vendors, or site engineers. As well as deliberate attacks, cyber vulnerabilities in SCADA systems may also be affected by inadvertent events (e.g., user errors, negligence equipment failures, and natural disasters). Security for protecting the entire Smart Grid technological environment requires the consideration of many subsystems that make up the Smart Grid, for example Wide Area Monitoring Protection and Control (WAMPAC), Distribution Management System (DMS), Advanced Metering Infrastructure (AMI), and higher level communication architectures at the grid system level. The scope of this paper is to focus on one important subsystem level of the Smart Grid environment, specifically cybersecurity for digital substations. This paper proposes a multilayer SCADA cyber-security attack detection system that improves Intrusion Detection System (IDS) technology. A realistic SCADA-specific cyber-security test-bed was also developed to investigate cyber attacks and to test the proposed IDS methods. This environment provides a platform for the indepth analysis of real attack scenarios in a replicated substation Local Area Network (LAN), in order to facilitate the development of effective attack countermeasure tools and technologies for the SCADA cyber domain.

Section II presents the related work. Section III proposes a conceptual multilayer cyber-security framework for SCADA 
systems. Section IV proposes a SCADA-specific IDS combining whitelist and behaviour-based methods. Section V discusses the implementation approach of the SCADA-IDS. In Section VI, a SCADA-specific cyber-security test-bed that investigates cyber attacks is presented to exemplify and validate the proposed SCADA-IDS. Section VII and Section VIII are discussion and conclusion, respectively.

\section{RELATED WORK}

SCADA systems in the Smart Grid will inevitably contain legacy systems that cannot be updated, patched, or protected by conventional IT security techniques. With limited computing resources in legacy devices and the lack of inbuilt security for SCADA systems, it is difficult to embed traditional cyber security techniques into these legacy systems. In these situations, new intrusion detection systems are needed to monitor the operation of such systems and to detect threats against the systems resulting from misuse by legitimate users or intentional attacks by external hackers.

Intrusion detection technologies in the IT domain are relatively mature and numerous intrusion detection methods have been presented [2]. Zhang et al. [3] present a distributed IDS for wireless mesh networks in Smart Grids, however this work does not directly relate to SCADA environments. Much research has been proposed and applied in intrusion and anomaly detection approaches targeted for SCADA systems, such as statistics based intrusion detection methods and SCADA-specific intrusion detection approaches [4-12]. However, research in SCADA specific detection tools is still at an early stage.

IDSs have been introduced to SCADA systems using statistical approaches to classify network traffic as normal or abnormal. To build the statistical models, various modelling methods can be used, such as neural networks, regression models, and Bayesian networks [9]. However, most statistical intrusion methods generate false positives which result in false alerts, and false negatives which miss real attacks.

SCADA-specific IDSs have been developed for SCADA systems using critical state, model and rule based methods. The primary limitation of current SCADA-specific IDSs is limited understanding of the range of SCADA applications and protocols, as highlighted by Idaho National Laboratory [4] Carcano et al. [6] propose a critical state-based IDS for SCADA based on the Modbus protocol in a power plant. However, this system can only detect a limited class of attacks against PLC systems. Model-based detection is not new in traditional IDS work, e.g., specification-based intrusion detection can be seen as model based. Cheung et al. [7] believe that model-based monitoring to detect unknown attacks is more feasible in SCADA systems than in general IT networks: three model-based techniques to monitor Modbus Transmission Control Protocol (TCP) networks, using protocol-level modes, communication-pattern-based detection and learning-based approach. Unfortunately, no quantitative results were obtained from this work nor detailed analysis regarding experimental validation. A rule-based IDS for an Intelligent Electronic Device (IED) based on IEC 61850 is realised by Snort in [8]. The Snort rules are obtained from experimental data based upon simulated cyber attacks, such as a Denial of Service (DoS) attack, password cracking, and Address Resolution Protocol (ARP) spoofing. The proposed blacklist approach is shown to detect known attacks effectively. However, blacklists are typically not effective against unknown threats or undiscovered vulnerabilities, also called zero-day attacks.

\section{Multilayer SCADA CYBER-SECURITY FrAMEWORK}

Current security countermeasures in SCADA systems mainly focus on protecting systems from external intrusions or malicious attacks. For example, incoming traffic to substations, control centres, and corporate networks will be inspected by commercial firewalls or IDSs. However, this security approach only considers perimeter defences and ignores interior detection within a substation network or a control centre. For instance, an engineer can enter a substation and connect his laptop to the LAN. An intentional or unintended attack via an infected laptop now has an improved chance of success because perimeter defences have been bypassed. In practice and in worst-case scenarios, all the cyber assets in SCADA systems should be regarded asvulnerable. However, we cannot demand that all the cyber assets meet the highest security requirements due to financial cost, time and system constraints. Therefore, in order to address this problem, a SCADA cyber-security framework based on SCADA-IDS is proposed, as illustrated in Fig. 1 that includes the following three aspects:

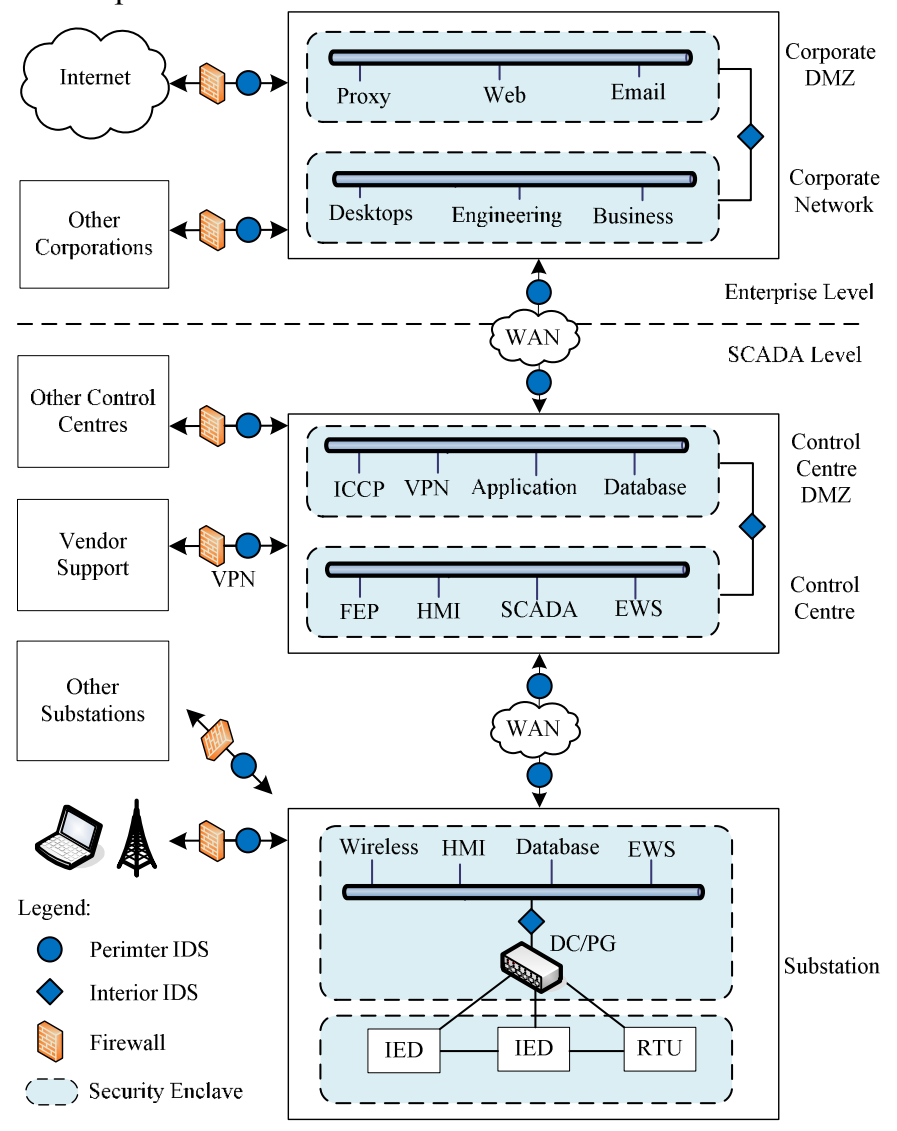

Fig. 1. Multilayer SCADA cyber-security framework with IDS 


\section{A. Security Enclaves}

A security enclave [13] is a secure group of cyber systems connected by one or more internal or external networks using suitable security policies and techniques in order to minimise the attack surface and its impact. It may be defined by logic functions or by physical distance. Compared with the traditional SCADA structure, the proposed secure architecture divides the normal corporate network into a new corporate network including enterprise servers (e.g., proxy, web and Email server) and corporate Demilitarized Zones (DMZ) involving desktops, laptops, Engineering Workstations (EWS), business servers, etc. In addition, the proposed secure architecture defines two enclaves in the control centre, i.e., the control centre DMZ containing the Inter-control Centre Communication Protocol (ICCP) sever, Virtual Private Network (VPN) server, database, etc and the control centre enclave including the Front End Processor (FEP), Human Machine Interface (HMI), SCADA/Energy Management System (EMS), etc, and two enclaves in the substation, as shown in Fig. 1. Here, DMZ means that a network segment as a "security buffer area" between the internal network and the external network. In the substation, the Data Concentrator (DC) or Protocol Gateway (PG) is used to collect and translate data from different IEDs or Remote Terminal Units (RTUs) with individual protocols.

\section{B. Perimeter Defence and Interior Detection}

The proposed enclave-based SCADA cyber-security framework focuses on perimeter defences against attacks from outside the enclaves and internal detection for malicious behaviours or misuse of employees from inside enclaves using the proposed multilayer SCADA-IDS scheme. In order to deploy appropriate perimeter defences in suitable locations, it is necessary to identify the boundaries of security enclaves. In Fig. 1 the SCADA-IDSs are deployed in the enclave boundaries for the perimeter defence, as well as inside the enclave for interior detection. A SCADA IDS can analyse traffic not only across enclave perimeters, but also within a security enclave, e.g., between an HMI and a PG in a substation.

\section{SCADA-IDS Management System}

The proposed SCADA-IDS management system contains Security Information and Event Management (SIEM) tools in the Security Operations Centre (SOC), IDS security managers at enterprise level and SCADA level, and distributed IDSs, as shown in Fig. 2. The SOC may include the correlation and intelligence capabilities to manage large-scale cyber incidents [21]. An SIEM (e.g., QRadar SIEM [20]) platform supports $\log$ management, real-time monitoring and security event management from a broad range of systems. It establishes an early warning system to detect threats based on log events and flow information from both the enterprise level and the SCADA level. The IDS security manager is designed to administer, monitor and configure an individual IDS by secure TCP/IP connections. It is possible that the Intrusion Detection Exchange Protocol (IDXP) is adopted to exchange information among different IDSs. Under real circumstances, a SCADA-
IDS can be set to a local mode which provides local security detection and log management; in addition, it transmits some data to a security manager for more comprehensive situational awareness across multiple security enclaves. Both commercial IDSs and the customized IDS can be adopted in the proposed SCADA cyber-security framework.

In this paper, a multi-attribute intrusion detection approach is proposed which is tailored for cyber-security at SCADA level, as described in the next section. The IDS system at enterprise level can be realised by commercial solutions, which is beyond the scope of this paper.

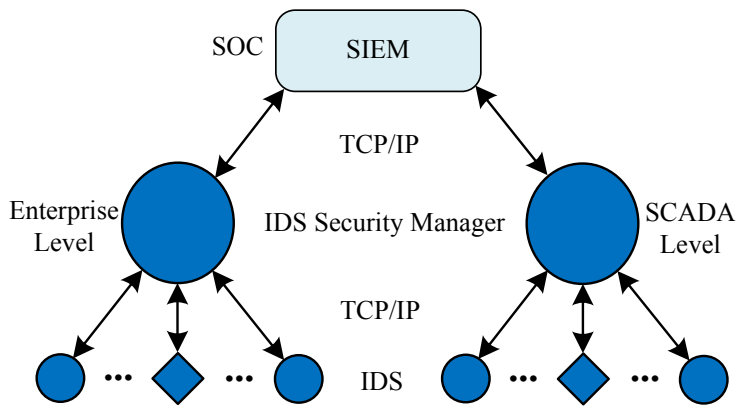

Fig. 2. SCADA-IDS security management system

\section{Proposed Multi-Attribute IDS For SCADA}

In comparison with traditional IT networks, SCADA systems have distinguishing features such as the use of a limited number of packets (low throughput), a fixed number of communication devices, a limited number of communication protocols, and regular communication and behaviour patterns. Therefore a SCADA-specific IDS is proposed as an effective tool to identify both external malicious attacks and internal unintended misuse. The proposed hybrid intrusion detection method consists of three attributes: i) Access control whitelists; ii) Protocol-based whitelists; iii) Behaviour-based rules. The basic detection procedure is illustrated in Fig. 3.

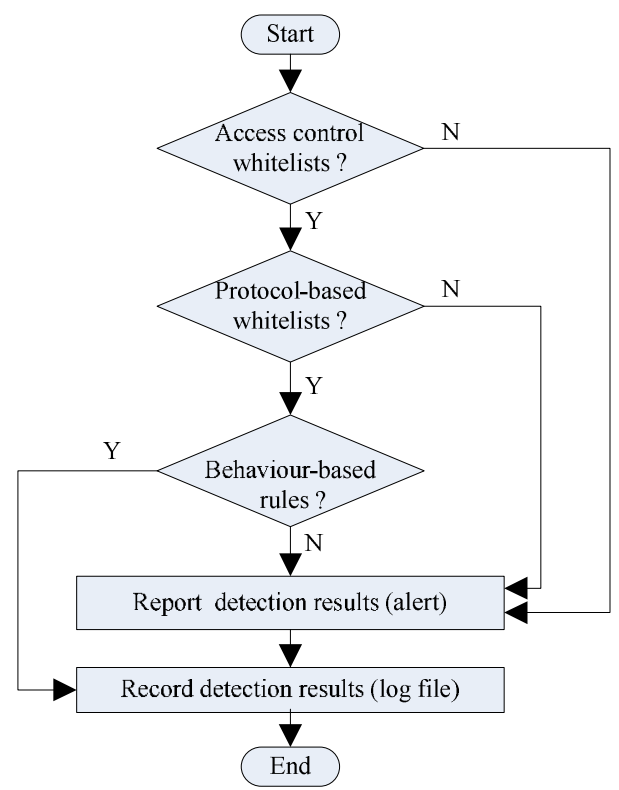

Fig. 3. Progress for Hybrid SCADA-IDS 


\section{A. Access Control Whitelists (ACW)}

The access control whitelist approach contains detectors in three layers, i.e., source and destination Medium Access Control (MAC) addresses $\left(M A C_{s r c}\right.$ and $\left.M A C_{d s t}\right)$ in the Ethernet layer, source and destination Internet Protocol (IP) addresses $\left(I P_{s r c}\right.$ and $\left.I P_{d s t}\right)$ in the network layer, and source and destination ports (Port src $_{\text {and }}$ and Pot $\left._{d s t}\right)$ in the transport layer. If any of the addresses or ports is not in the corresponding whitelist, the detector will take a predefined action, e.g., alert in IDS mode and log the detection results. That is,

$$
A C \notin\left\{A C_{w l}\right\} \rightarrow \text { Actions(alert,log) }
$$

where $A C=M A C_{s r c}, M A C_{d s t}, I P_{s r c}, I P_{d s t}$, Port $_{s r c}$, Port $_{d s t}$ and $A C_{w l}$ represents corresponding whitelist set.

In addition, each host or device in a SCADA system has a unique $<I P, M A C>$ match. If the device has not been replaced with new hardware and the same IP address of the device is detected from two or more MAC addresses, it means that a spoofing attack may be happening.

\section{B. Protocol-Based Whitelists (PBW)}

The aforementioned access control whitelist refers to layer 2, 3 and 4 in terms of the Open Systems Interconnection (OSI) model. The protocol-based whitelist method is related to the application layer (up to layer 7) and deals with various SCADA protocols such as Modbus, DNP3, IEC 60870-5 series, ICCP, IEC 61850, and proprietary protocols. In different scenarios, the detector can be set to support specific protocols. For example, when the IDS is deployed at the network between two control centres, the protocol-based detector only allows communication traffic complying with specific protocols, otherwise it will generate an alert message.

\section{Behaviour-Based Rules (BBR)}

As a necessary complement to the aforementioned whitelist methods, a behaviour-based detection approach finds and defines normal and correct behaviours by Deep Packet Inspection (DPI). This may include analysis of a single-packet or multiple-packet together. SCADA-IDS in different scenarios may have different rules in terms of normal behaviours. If the IDS is located between a HMI and a protocol gateway within a substation, several behaviour-based detectors are proposed and defined as follows.

1) Correlation Detector: For a specific switching device, the switching state correlates with relevant measured values. For instance, if the switching state changes between open and closed, relevant measure values will correctly vary, otherwise, alarms will occur, i.e.,

$$
\left.\begin{array}{l}
\text { If }(S V=\text { open }) M V(I)>e_{o} \\
\text { If }(S V=\text { closed }) M V(I)<e_{c}
\end{array}\right\} \rightarrow\left\{\begin{array}{c}
\text { Alert } \\
\text { Log }
\end{array}\right.
$$

where $S V$ represents a switching value; $M V(I)$ means measured current values; $e_{o}$ or $e_{c}$ is the positive threshold of the electric current value which is near zero.

2) Relay Protection Function Detector: IED relay equipment generally has multiple protection functions (such as over-load, over-current, and instantaneous over-current) for the purposes of detecting faults and minimizing impacts of faults by tripping the associated circuit breakers in power systems. When an IED detects a fault and takes some actions according to the associated protection algorithm, the alarm or trip information will be sent to the HMI in a substation or a control centre by remote signalling data. The detector utilises correlated information from remote measurement data to detect whether the protection information is correct or not. For example, in terms of the over-load protection, provided one of three-phrase currents exceeds a certain value for a specified period of time, the over-load protection action will occur. Meanwhile, the alarm or trip information will be uploaded as follows.

- Over-load alarm: When an over-load alarm signal happens, at least one of the associated current measurement values should exceed the predefined over-load protection setting value. In contrast, when the over-load alarm signal disappears, three-phrase current measured values are all below the setting value. If any of the two rules is violated, the detector will generate actions. i.e.,

$$
\left.\begin{array}{l}
\text { If }\left(R S_{\text {ola }}=1\right) M V\left(I_{a}, I_{b}, I_{c}\right)<I_{\text {ol }} \\
\text { If }\left(R S_{\text {ola }}=0\right) M V\left(I_{a}\left|I_{b}\right| I_{c}\right)>I_{\text {ol }}
\end{array}\right\} \rightarrow\left\{\begin{array}{c}
\text { Alert } \\
\text { Log }
\end{array}\right.
$$

where $R S_{\text {ola }}=1,0$ means the over-load alarm signal happens and disappears, respectively; $M V\left(I_{a}, I_{b}, I_{c}\right)$ and $M V\left(I_{a}\left|I_{b}\right| I_{c}\right)$ represent all the three-phrase current measured values and one of the three-phrase current measured values, respectively; $I_{o l}$ is over-load protection setting value.

- Over-load trip: When an over-load trip signal happens, all the three-phrase current measured values should be near zero. In contrast, when the over-load trip signal disappears, all the three-phrase current measured values will be below the setting value. If any of the two rules is violated, the detector will act. i.e.,

$$
\left.\begin{array}{l}
\text { If }\left(R S_{o l}=1\right) M V\left(I_{a}\left|I_{b}\right| I_{c}\right)>e_{0} \\
\text { If }\left(R S_{o l}=0\right) M V\left(I_{a}\left|I_{b}\right| I_{c}\right)>I_{o l}
\end{array}\right\} \rightarrow\left\{\begin{array}{c}
\text { Alert } \\
\text { Log }
\end{array}\right.
$$

where $R S_{o l}=1,0$ means that the over-load trip signal happens and disappears, respectively; $M V\left(I_{a}\left|I_{b}\right| I_{c}\right)$ means one of the three-phrase current measured values; $e_{o}$ represents a positive current value which is close zero; $I_{o l}$ is over-load protection setting value.

3) Time-Related Detector: If the control commands are not correctly executed due to cyber attacks or misuse, a power network may become insecure or potentially unstable. Critical control commands have time-related constraints such as time interval limit and frequency limit. If the same command is sent too frequently, it may violate the following rules. In each case the detector will initiate some actions (alert and log).

$$
C V(n)-C V(n-1)<T \rightarrow \text { Actions (alert, } \log )
$$

where $C V$ is a control command; $n$ is a positive integer $(n>1)$; $T$ is the limit of time interval.

$$
\frac{C V(n)-C V(1)}{n-1}>F \rightarrow \text { Actions (alert, log) }
$$

where $F$ represents the frequency limit.

4) Length Detector: When a SCADA packet contains bytes which indicate the length information about the packet in the 
payload, it is proposed that a length detector should be applied to detect that whether the number shown in the length bytes equals to real length of the payload, such that,

$$
P L_{l} \neq P L_{r l} \rightarrow \text { Actions (alert, log) }
$$

where $P L_{l}$ is the length value indicated in the length field of the payload, and $P L_{r l}$ stands for practical length of the payload.

5) Range Detector: Normally, measured values belong to an operational range with upper and lower boundary values. Such measured values may include current $(I)$, voltage $(U)$, active power $(P)$, reactive power $(Q)$, and frequency $(f)$. If the measured value is outside the expected range, some actions will execute automatically, i.e.,

$$
\begin{aligned}
& M V(i) \notin\left[M V(i)_{\min }-e(i), M V(i)_{\max }+e(i)\right] \\
& \rightarrow \text { Actions }(\text { alert }, \log )(i=I, U, P, Q, f, \ldots)
\end{aligned}
$$

where $M V(i)(i=I, U, P, Q, f, \ldots)$ represents different measured values such as current, voltage, active power, reactive power, and frequency, $\left[M V(i)_{\min }-e(i), M V(i)_{\max }+e(i)\right]$ stands for the range between the upper and lower boundary and $e(i)$ measures the tolerance.

6) Function Code Detector: In terms of industrial network protocols, one of the common features is the use of function codes (used in DNP3) or type identification (used in IEC 60870-5 series). The function code (or type identification) detector only allows specifically defined function codes (or type identification) according to different SCADA protocols, or else security actions will occur. Using the function code detector as an example,

$$
P L_{f_{c}} \notin\left\{F C_{i} \mid i=1,2, \ldots, n\right\} \rightarrow \text { Actions (alert,log) }
$$

where $P L_{f c}$ is a function code in the payload and $F C_{i}$ represents the allowed function codes based on protocols.

\section{SCADA-IDS IMPLEMENTATION}

In order to implement the SCADA-specific IDS proposed in this paper, a SCADA-IDS based on the Internet Traffic and Content Analysis (ITACA) tool is developed. ITACA [14] is a software platform for traffic sniffing and real-time IP network analysis which has been developed by the Centre for Secure Information Technologies (CSIT) at the Queen's University of Belfast. The extendable analysis tool enables the implementation of plugins to perform specific tasks, e.g., IDS. In this paper, the SCADA-specific IDS is developed in $\mathrm{C} / \mathrm{C}++$ using the ITACA platform, as illustrated in Fig. 4.

The real-time SCADA-IDS combines ACW, PBW and $\mathrm{BBR}$, as presented in Section IV, based on DPI including single-packet and multiple-packet inspection. In the initialisation stage, the parameters of SCADA-IDS are preset. The detailed implementation steps are as follows.

1) The raw bytes of packet data are captured from the SCADA network by a network layer interface, which is realised by the Packet Capture (PCAP) library. The ITACA core can extract, interpret and analyse the SCADA flows and packets up to $4 \mathrm{~Gb} / \mathrm{sec}$ in order to provide all possible information for the realisation of SCADA-IDS plugins. It includes the following main modules: the protocol extractor, packet storage, flow look up table, event generator, plugin queues and event controller. The detailed modules of the ITACA core architecture are described in [14].

2) To realise the ACW introduced in Section IV-A, the trusted source and destination MAC addresses, IP addresses and ports in the SCADA network are preset in the initialisation stage.
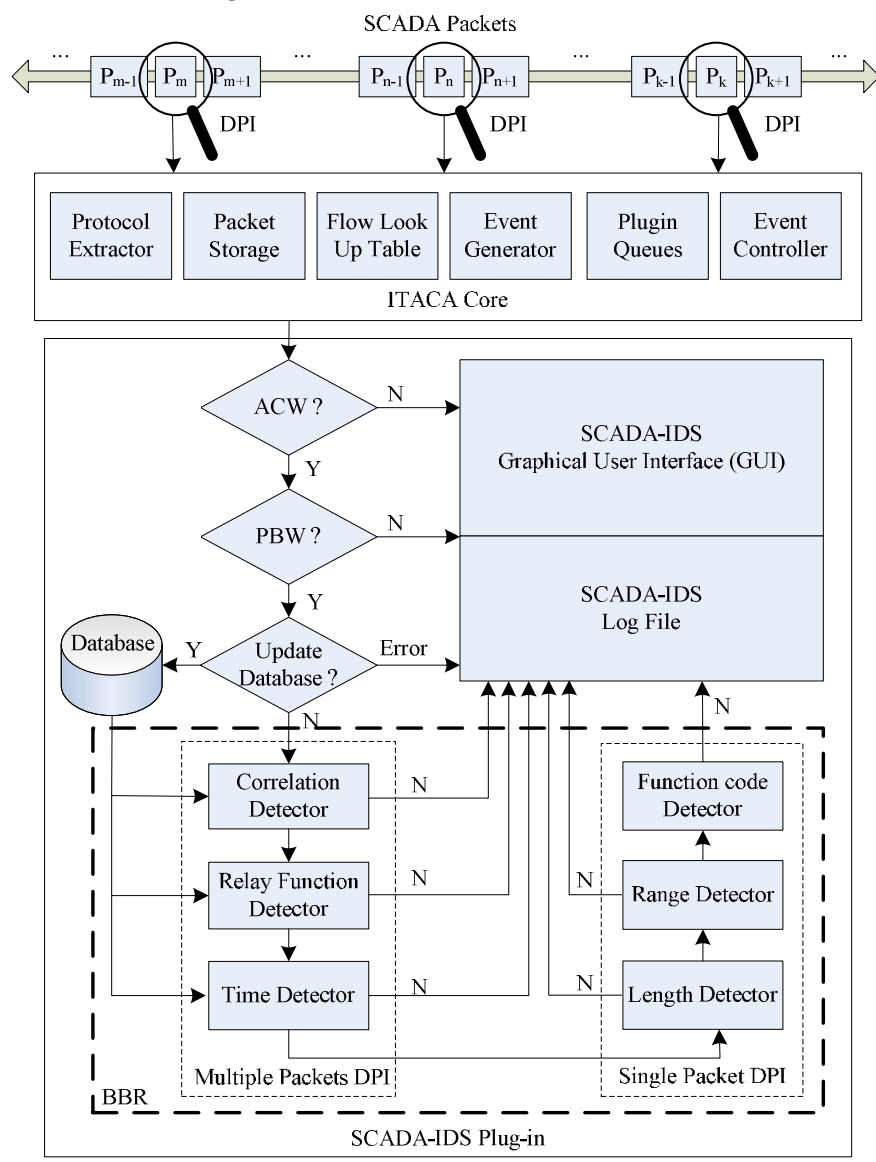

Fig. 4. The process for the implement of proposed SCADA-IDS

3) To implement the PBW discussed in Section IV-B, the Perl Compatible Regular Expressions (PCRE) library is utilised to identify the SCADA protocol based on application layer data using regular expression pattern matching. The SCADA protocol type is determined in the initialisation stage according to a specific application scenario. The proposed SCADA-IDS is capable of supporting widely used SCADA protocols such as Modbus, DNP3, IEC 60870-5-103/104, ICCP, IEC 61850, and some proprietary protocols.

4) A database is set up for the SCADA-IDS which stores critical status parameters of the SCADA system in order to realise multiple packets (cross-packet) inspection, for example, to determine the status of circuit breakers and protective relays. If the packet data has passed the detection of ACW and PBW, the database will be updated when the relevant status changes.

5) The following detectors belong to BBR as presented in Section IV-C. Among them, the time-related detector, correlation detector and relay function detector span multiple packets which need the support of the database. The other detectors are single packet inspection such as the length detector, function code detector, and range detector. 
6) In the correlation detector described in Section IV-C, the threshold values $e_{o}$ or $e_{c}$ are preset. In terms of the relay function detector, the over-load protection setting value $I_{o l}$ is set according to the specification of IED and the practical application. In the time-related detector mentioned in Section IV-C, the parameters $T$ and $F$ are set in the initialisation stage. The range parameters of the range detector are set in the initialisation stage. The function codes of the function code detector are also set according to a proprietary SCADA protocol.

If a packet violates any rule implemented above (e.g., ACW, PBW, or BBR), the SCADA-IDS will take the appropriate action (e.g., alert), record the detection results in the $\log$ file, and display the results in the Graphical User Interface (GUI), as shown in Fig. 4. The GUI is designed and developed using Glade and Gtkmm in order to display the detection performance and results.

\section{SCADA-SPECIFIC CYBER-SECURITY TEST-BED AND EXPERIMENTAL RESULTS}

This section presents a SCADA-specific cyber-security test-bed focusing on a security enclave within the substation. It can be used to investigate cyber-security vulnerabilities and implement proposed hybrid intrusion detection approaches in a SCADA system. The test-bed is based on a real gridconnected photovoltaic (PV) SCADA system that has been deployed in a practical environment, as illustrated in Fig. 5, which uses protocols based on IEC 60870-5 series.

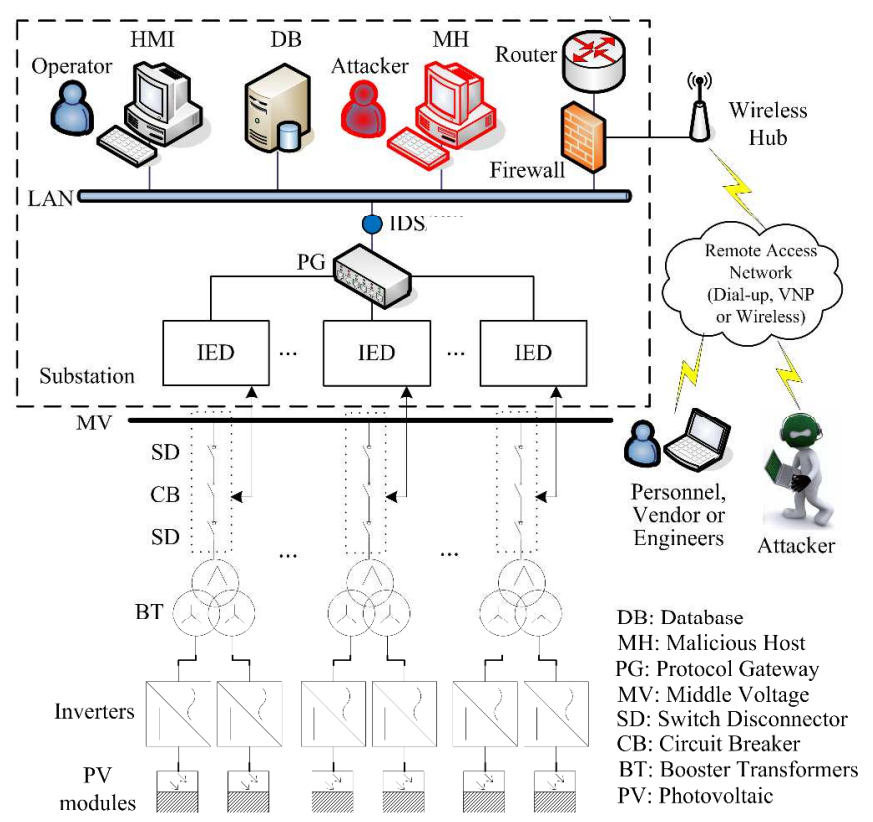

Fig. 5. SCADA cyber-security test-bed

\section{A. Test-Bed Architecture}

The test-bed architecture contains an HMI, database, malicious host (simulated attacker), IDS host, protocol gateway (PG), IED simulator (hereafter referred to as IED), switch, firewall, router etc, as shown in the dashed box of Fig. 5. Three Microsoft Windows-based hosts (HMI, PG, IED) simulate real-time SCADA communication in a substation LAN. The HMI host simulates the master station where Commercial Off-The-Shelf (COTS) SCADA supervisory control software is installed. The PG host with different COTS communication protocol gateway software is used to connect IEDs with the HMI. The HMI and PG are connected by a switch. The IED communicates with the PG using the IEC 60870-5-103 protocol. Due to confidentiality concerns the names of the SCADA software and the simulated IED in the test-bed are withheld.

The Linux-based malicious host is used to simulate a malware infected computer inside the LAN, or a laptop connected to the LAN from the outside (e.g., a maintenance access), which can be controlled by an attacker. Many cyber attacks can be investigated in the test-bed such as DoS, ARP spoofing, and Man-in-the-Middle (MITM) attacks.

For testing, the proposed SCADA-specific IDS is deployed between the HMI and PG as an interior detection tool. The SCADA-IDS is implemented based on the ITACA tool in the Linux-based host (see IDS in Fig. 5) which is connected to the LAN by port mirroring.

\section{B. Man-in-the-Middle Attack}

ARP is primarily used to resolve network layer addresses (IP addresses) into data link layer addresses (Ethernet MAC addresses) in LAN communication. The ARP spoofing attack is used to modify the cached $\angle I P, M A C>$ pairing in the local ARP cache table [15]. Such a MITM attack allows an attacker to sniff or tamper information in a LAN by ARP spoofing [16], [17].

In the test-bed environment presented in this paper, an ARP spoofing attack is launched by a Metasploit [18] module in Backtrack 5 which is Linux-based penetration testing software. This approach is used as it is straightforward to perform for testing purposes. Other more complex "MITM" attacks may be caused by malware, resulting in similar behaviours in the network. ARP is a stateless and trusting protocol and does not provide any verification mechanism to verify the authenticity of the ARP requests and replies, so attacks are possible from malicious hosts in a LAN. In the ARP cache poisoning attack launched by Metasploit, the attacker $(\mathrm{MH})$ sends ARP replies to the PG host indicating that HMI host with the IP **.100.100.98 has the MAC $* *$ **:27:ed:09:0f which is the MAC address of the attacker, so the PG host will update its ARP cache table with the $<* * .100 .100 .98, * * * *: 27: e d: 09: 0 f>$ paring. In this case, the attacker impersonates the HMI so that the PG host will send packets destined to the HMI to the attacker instead.

Similarly, the HMI host can also become the target host of a spoofing attack. After local ARP cache in the HMI is poisoned, the $<I P, M A C>$ pairing in the ARP cache table will be updated from <**.100.100.80, **:**:43:bb:74:4a> to $<* * .100 .100 .80$, **:**:27:ed:09:0f>

Furthermore, by poisoning the HMI host and the PG host at the same time, the attacker can silently stay in the middle of the two hosts (HMI and PG) to launch a MITM attack in the test-bed in order to easily sniff all the traffic sent in both directions and inject new data into both. The malicious 
attacker may utilise the intercepted information to launch more severe attacks later.

In the MITM attack experiment, an attack simulator is developed using $\mathrm{C} / \mathrm{C}++$ in order to send modified information to the HMI host or the PG host. The injected malicious data from the attacker will display on the screen of the HMI host which may mislead the operator. In a worse-case context a false remote operation command such as "open the circuit breaker" from the attacker could shed the PV grid and affect power supply reliability, and perhaps threaten safety.

\section{SCADA-IDS Experiment and Results}

For the SCADA-IDS experiment, test network traffic was generated which included normal and malicious packets which may be the goal of a MITM attack. The normal SCADA traffic between the HMI and the PG was captured by the SCADA-IDS host which is connected to the LAN via port mirroring, as shown in Fig. 5. Then, abnormal packets were introduced into the test dataset by a MITM attack experiment in order to verify proposed whitelist and behaviour based attack detection approaches. In this experiment, 500 packets are captured including $50(10 \%)$ simulated abnormal packets, and wherein the number of abnormal packets violating $\mathrm{ACW}$, PBW and BBR is $12(2.4 \%), 7(1.4 \%)$ and $31(6.2 \%)$, respectively. It can be seen from the experimental results that the proposed SCADA-IDS can effectively identify all the abnormal data without false positives for the given experiment, as shown in Fig. 6.

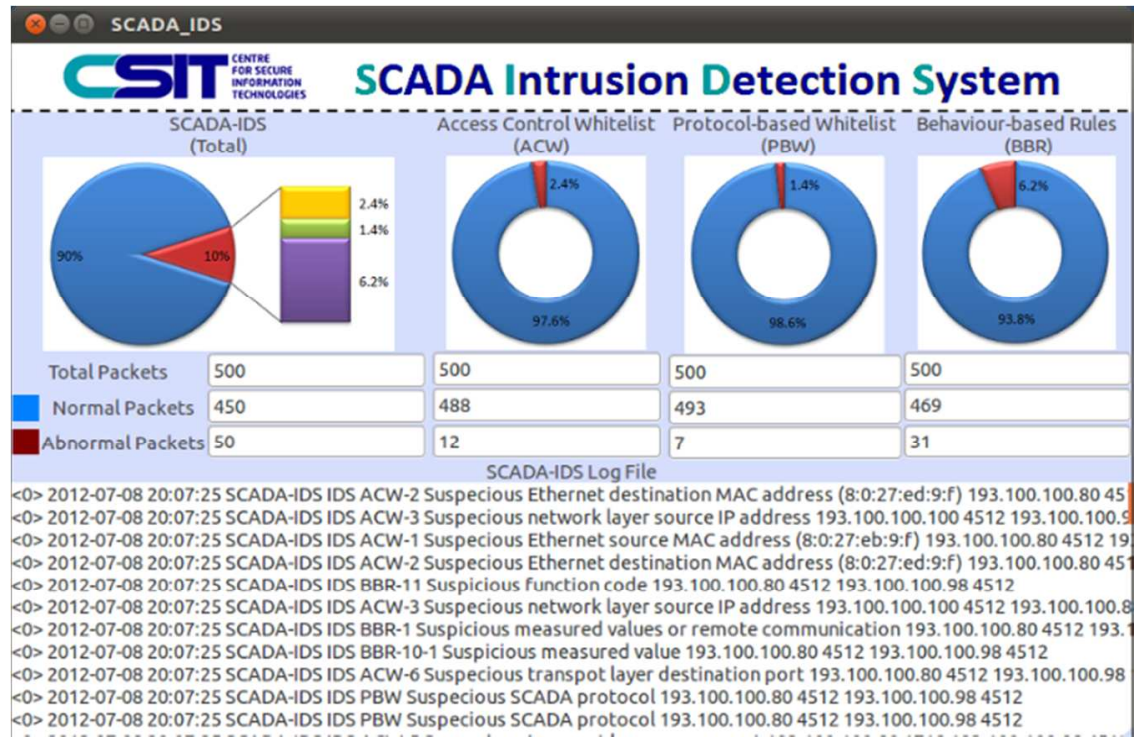

Fig. 6. The GUI for displaying SCADA-IDS detection results

The SCADA-IDS records all the detection results in a log file and displays in the GUI (Fig. 6). The log file is defined referring to RFC 3164 . The detailed message format is as follows:

<SEVERITY> TIMESTAMP DEVICE NAME DEVICE TYPE ALERT TYPE EVENT_DESCRIPTION ${ }^{-}$SRC IP SRC PORT DST I $\bar{P}$ DST PORT

In this case, SEVERITY represents alert severity which is described by a numerical code, e.g., 0, 1, 2 and 3 stand for EMERGENCY, ERROR, WARNING and NOTICE, respectively. The TIMESTAMP field is the local time and is in the format of "YYYY-MM-DD HH:MM:SS". DEVICE NAME means the name or IP address of specific security device. DEVICE TYPE is the type of the security device, e.g., IDS. ALERT TYPE represents alert event type which is violated such as $A C W, P B W$, or BBR. EVENT DESCRIPTION describes the detailed information of the specific security event. SRC IP, SRC PORT, DST IP and DST PORT are source IP address, source port, destination IP address and destination port, respectively.

The log messages generated as an output from this experiment are explained in detail as follows. Fig. 7 shows an alert that suspicious Ethernet destination MAC address is detected when the packet is sent from PG host (**.100.100.80) to HMI host $(* * .100 .100 .98)$. In the alert resulting from an ARP spoofing attack, one of ACWs is violated (discussed in Section IV-A).

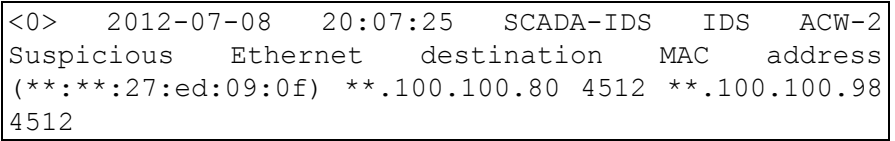

Fig. 7. The ACW alert message in the log file

In Fig. 8, the suspicious SCADA protocol is detected by PBW, which verifies the proposed protocol based whitelist approach as presented in Section IV-B. Any cyber attack which violates the SCADA protocol specification will be alerted.

\begin{tabular}{|llllll|}
\hline$<0\rangle$ & $2012-07-08$ & $20: 07: 25$ & SCADA-IDS & IDS PBW Suspicious \\
SCADA protocol & $\star \star .100 .100 .80$ & 4512 & $\star *$ & .100 .100 .98 & 4512
\end{tabular}

Fig. 8. The PBW alert message in the log file

Fig. 9 illustrates part of the alert messages generated due to the BBR violation (described in Section IV-C). For example, $\mathrm{BBR}-1, \mathrm{BBR}-2, \mathrm{BBR}-4, \mathrm{BBR}-8, \mathrm{BBR}-10-1$ and $\mathrm{BBR}-11$ 
specifically refer to the correlation detector, relay function detector, time-related detector, length detector, range detector and function code detector, respectively. The results show how this behaviour based approach can be effective against zero-day attacks, since the physical effects are also detected, rather than only the IT causes.

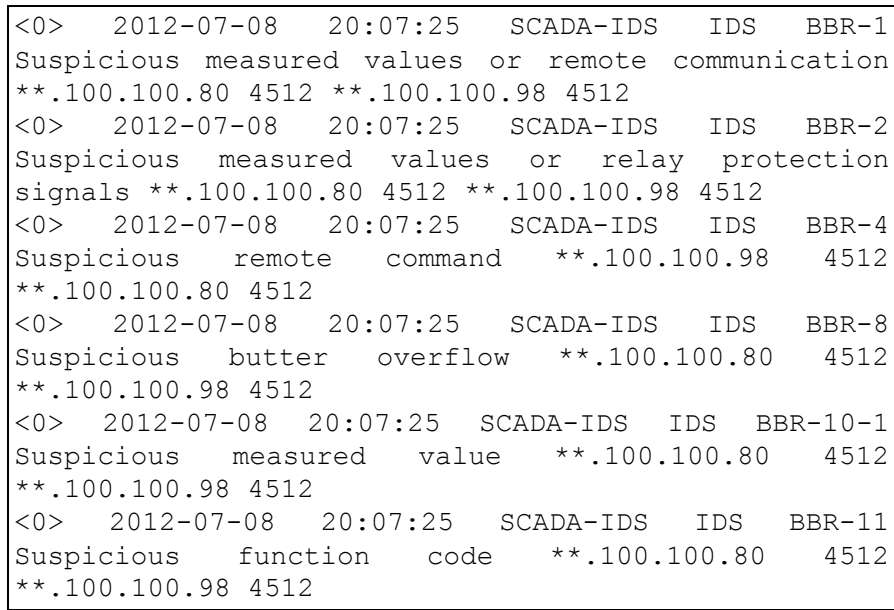

Fig. 9. The BBR alert messages in the log file

\section{Maximum Execution Time Estimate}

To guarantee reliable operation in SCADA-based control systems in power systems, latency is a critical issue for communications. Thus, it is necessary to consider the latency introduced by any cyber-security process. A statistical estimation model using Gumbel distribution in [22] is adopted to predict an extreme execution time based on execution time samples obtained by experiments. The Gumbel distribution belongs to the extreme value distribution family, which has a cumulative distribution function representing the likelihood that the maximum of a set of sample data of the form $\left\{x_{1}, \ldots\right.$, $\left.x_{n}\right\}$ will be equal to, or less than, $x$. The Gumbel distribution function is as follows:

$$
G_{(\lambda, \delta)}(x)=\exp \left\{-\exp \left(\frac{-(x-\lambda)}{\delta}\right)\right\}, x>\lambda
$$

where $\lambda$ and $\delta$ are location and scale parameters, which can be estimated by maximum likelihood estimation (detailed information is in [22]).

Equation (10) may give the estimated value less than the largest piece of sample data. It is necessary for the estimation of maximum execution time to only consider values greater than the largest value of sample data denoted by $\max _{i}$. Considering this constraint, the Gumbel distribution is as follows:

$$
\begin{array}{r}
\theta_{i(\lambda, \delta)}(x)=G_{(\lambda, \delta)}^{\max _{i}}(x)=P\left(X \leq x \mid X>\max _{i}\right)= \\
\frac{P\left(\max _{i}<X \leq x\right)}{P\left(X>\max _{i}\right)}=\frac{G(x)-G\left(\max _{i}\right)}{1-G\left(\max _{i}\right)}
\end{array}
$$

The estimation of the maximum execution time is derived from (11). For any estimate $\omega_{i}$ the probability that the most extreme execution time will occur at, or below, this value will be based on the estimation model, as shown in (12).

$$
G_{\left(\lambda_{i}, \delta_{i}\right)}^{\left(\max _{i}\right)}\left(\omega_{i}\right)=1-\eta_{i}=\varphi_{i}
$$

where $\eta_{i}$ is the likelihood at which an estimate of the maximum execution time is exceeded, and $\varphi_{i}$ is corresponding confidence level.

In this experiment, the SCADA-IDS execution environment uses an Ubuntu 11.04 64-bit operation system running on a quad-core Intel $\mathrm{i} 7$ processor using a $\mathrm{g}++4.5 .2$ compiler. This experiment was repeated 60 times, with a maximum execution time $\max =59 \mu \mathrm{s}$, a sample mean of 46.5 $\mu$ s and a standard variance of 24.8. The scale parameter $\delta$ and the location parameter $\lambda$ is 19.34 and 35.34, respectively. Therefore, the estimation model of the maximum execution time for the SCADA-IDS experiment based on (11) and (12) is given as:

$\theta_{(\lambda, \delta)}(x)=\varphi=-2.92+3.92 \exp (-\exp (-0.0517 x+1.827))$

From (13), it is possible to evaluate the confidence with different estimate values for maximum execution time, as shown in Fig. 10.

From the above statistical analysis, it can be seen that the estimated maximum execution time of the SCADA-IDS is less than or equal to $151 \mu$ s with $99 \%$ confidence (Fig. 10) and less than or equal to $254 \mu$ s with $100 \%$ confidence, which would not compromise timely availability of data for normal operation of SCADA systems. According to IEEE standards for electric power substation automation [19], high-speed protection information data delivery time requirements are less than $1 / 4$ cycle ( $5 \mathrm{~ms}$ in $50 \mathrm{~Hz}$ systems). Clearly the latency of the SCADA-IDS meets the specified time requirement of electricity control systems.

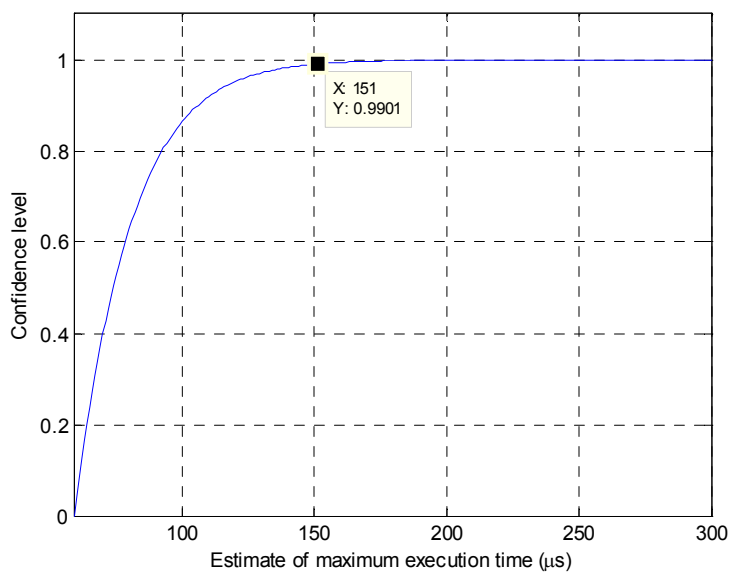

Fig. 10. The diagram of confidence level against maximum execution time estimate

\section{DISCUSSION}

According to the aforementioned experiments and results, it is clear that the proposed multi-attribute SCADA-IDS is an effective tool for early warning, detection and prevention of intrusion and abnormal behaviours in evolving SCADA which will support power system automation.

The statistical IDS [9] applied to SCADA systems adopts statistical approaches such as neural networks and Bayesian 
methods to distinguish the abnormal data from the normal traffic. However, these methods may lead to false positives and false negatives which inevitably will result in false alarms and missed attacks. Therefore, although such techniques have some merits, when used alone they are not sufficiently accurate. This is partly why a multi-attribute approach is preferable.

TABLE I

SCADA-Specific IDS Comparisons

\begin{tabular}{|c|c|c|c|c|c|c|}
\hline IDS & Application scenarios & Protocols & $\begin{array}{l}\text { Implementation } \\
\text { methods }\end{array}$ & $\begin{array}{l}\text { Implementation } \\
\text { tool }\end{array}$ & Process time & Accuracy \\
\hline$[6]$ & Power plants & Modbus TCP & Critical state analysis & $\mathrm{C} \#$ & $<1 \mathrm{~ms}$ & $99 \%$ \\
\hline$[7]$ & $\begin{array}{l}\text { Process control } \\
\text { systems }\end{array}$ & Modbus TCP & Model-based detection & Snort & Not published & Not published \\
\hline$[8]$ & IEC 61850 substations & $\begin{array}{c}\text { ARP/ICMP/HTTP/ } \\
\text { FTP/Telnet }\end{array}$ & Blacklist rules & Snort & Not published & $100 \%$ \\
\hline [23] & $\begin{array}{l}\text { Some SCADA } \\
\text { systems }\end{array}$ & Modbus/DNP3 & State-based detection & $\mathrm{C \#}$ & Not published & $100 \%{ }^{*}$ \\
\hline $\begin{array}{l}\text { Proposed } \\
\text { SCADA- } \\
\quad \text { IDS }\end{array}$ & Digital substations & $\begin{array}{c}\text { IEC } 60870-5 \text { series/ } \\
\text { DNP3/proprietary } \\
\text { protocols }\end{array}$ & $\begin{array}{c}\text { Whitelist and behaviour } \\
\text { based approaches } \\
\text { (ACW+PBW+BBR) }\end{array}$ & $\begin{array}{l}\text { ITACA } \\
(\mathrm{C} / \mathrm{C}++)\end{array}$ & $<254 \mu \mathrm{s}$ & $100 \%$ \\
\hline
\end{tabular}

Note: * The accuracy is $100 \%$ under data rates of $180 \mathrm{~kb} / \mathrm{s}$.
First, the proposed SCADA-IDS provides a wider compatibility in terms of application scenarios and protocols handled, e.g., SCADA protocols in digital substations, such as IEC 60870-5 series, DNP3, and proprietary protocols. In comparison, [6] and [7] only support Modbus TCP in power plants and process control systems, respectively. The Snort rules in [8] refer to ARP, Internet Control Message Protocol (ICMP), Hypertext Transfer Protocol (HTTP), File Transfer Protocol (FTP), Telnet, rather than SCADA protocols themselves. The proposed IDS also extends the attack scenario detection abilities in [8], namely, MITM against SCADA protocols.

Compared with the proposed multi-attribute IDS implementation on ITACA, [8] uses blacklist rules in Snort parlance, which are not effective against unknown attack. In addition, the proposed IDS implementation has better flexibility than Snort. This is because it is built using ITACA which provides database capabilities to implement userdefined detection strategies, such as correlation detector, relay function detector, and range detector. With Snort it is difficult to realise these behaviour-based rules.

The process time is a critical property for evaluating SCADA-IDS performance; however, unfortunately, [7], [8] and [23] do not provide evident IDS execution times. According to the statistical estimation in Section VI-D, the maximum execution time will be less than or equal to $254 \mu \mathrm{s}$ with $100 \%$ confidence, which is better than [6]. In terms of the IDS accuracy, because deterministic detection approaches are presented, rather than statistical or pattern recognition algorithms [8], the proposed IDS will consequently detect all the malicious packets in any given experiment.

Compared with the previous IDS methods, the novel approach proposed here firstly applies whitelist and behaviour based IDS to SCADA systems combining knowledge of power systems (domain knowledge) with network security
Setting aside the statistical approach, a comparison will now be considered between the proposed IDS and the most relevant state-of-the-art proposals. Although it is difficult to directly compare different published technologies, which use different scenarios and protocols, some indirect and valid comparisons can be made, as shown in Table I. techniques. In particular, it is based on fully considering the operational features and most common protocols of SCADA systems. In addition, the proposed SCADA-IDS can effectively identify permitted and non-permitted devices, connections, and protocols with enhanced payload inspection functionality to detect permitted and non-permitted behaviours and operations. Therefore, the multi-attribute SCADA-specific IDS can be effective against not only known attacks but also unknown attacks. Moreover, it can deal with intrusions from outside electricity utilities, as well as inadvertent events from inside, in order to make cyber space in SCADA systems more secure. Furthermore, as it passively analyses data on the network, the susceptibility of the IDS itself to attacks is minimal. The proposed SCADA-IDS was implemented as a plugin in ITACA, and the flexible design architecture of ITACA ensures that the SCADA-IDS plugin provides sufficient throughput and low latency such that the practical communication requirements [19] of SCADA systems in power systems are met, as shown in Section VI-D.

In order to successfully deploy the proposed SCADA-IDS into a live real-world environment, careful consideration will need to be given to how the tool can be optimally configured during the initialisation stage. Security engineers installing tools in this domain must understand specific aspects of the SCADA systems to which the IDS will be deployed. Knowledge of the communication protocols, field device functions, and application environments, is also vital to ensure false positive or false negative alarms are minimised. It is advisable that initial tests be carried out on "mirrored" systems that exactly replicate the performance of the live SCADA system, in order to provide a robust verification stage that is not possible in the presented test-bed. Ongoing efforts will also be required in order to update the capabilities of the IDS to detect and mitigate emerging and evolving threats.

Finally, a significant challenge in this area of research is 
the lack of an openly available test dataset to compare the performance and accuracy of proposed solutions. This is understandable from the perspective of SCADA system operators, due to the sensitive nature of the data. However for research in the community to progress, such a dataset would be valuable.

\section{CONCLUSION}

This paper has presented a layered cyber-security framework for SCADA systems which combines security enclaves, IDS technology and behavioural monitoring to make SCADA systems more secure. The framework provides a hierarchical approach for an integrated security system, comprising distributed IDSs. This approach is compatible with currently emerging trends towards using SIEM technology to monitor Smart Grids and other critical infrastructure. In this context a novel SCADA-IDS with both whitelists and behaviour-based SCADA protocol analysis is proposed and exemplified in order to detect known and unknown cyber attacks from inside or outside of SCADA systems. Finally, the proposed SCADA-IDS is implemented and successfully validated through a series of realistic scenarios performed in a SCADA-specific test-bed developed to replicate cyber attacks against a substation LAN.

Digital substations are critical nodes that are integral to the core functions of electricity grids. Consequently, their dependable operation is essential to ensure power delivery remains secure, stable and reliable. In the context of the rapid development and deployment of digital substations around the world, timely research on emerging cyber-security issues in this area is a highly relevant and urgent issue. However, security the digital substation environment is just part of a wider and significant effort that is required to ensure the secure operation of advanced power systems. Many challenges remain to be addressed in other subsystems and for the higher level communications architecture where subsystems are interconnected.

Based on published knowledge of cyber vulnerabilities and attack scenarios, it is clear that a large number of viable cybersecurity issues exist against Smart Grid SCADA systems, which could threaten digital substations. To the best of authors' knowledge, and with reference to the discussion in Section VII, it is believe the proposed comprehensive approach and implemented SCADA-IDS presents a significant contribution to addressing emerging cyber-threats to digital substations, and the secure operation of the wider Smart Grid infrastructure.

\section{REFERENCES}

[1] Antiy CERT. (2010, Sep.). Report on the Worm Stuxnet's Attack. Antiy Corp., Harbin, China. [Online]. Available: http://www.antiy.net/en/analysts/Report_On_the_Attacking_of_Worm_Struxn et_by_antiy_labs.pdf

[2] A. A. Ghorbani, W. Lu. and M. Tavallaee, Network Intrusion Detection and Prevention: concepts and techniques. London: Springer, 2010, pp. 1-20.

[3] Z. Yichi, W. Lingfeng, S. Weiqing, R. C. Green, and M. Alam, "Distributed Intrusion Detection System in a Multi-Layer Network Architecture of Smart Grids," IEEE Trans. Smart Grid, vol. 2, pp. 796-808, Dec. 2011.
[4] J. Verba and M. Milvich, "Idaho National Laboratory Supervisory Control and Data Acquisition Intrusion Detection System (SCADA IDS)," in Proc. 2008 IEEE Conf. on Technologies for Homeland Security, pp. 469-473.

[5] M. P. Coutinho, G. Lambert-Torres, L. E. B. da Silva, H. G. Martins, H. Lazarek, et al, "Anomaly detection in power system control center critical infrastructures using rough classification algorithm," in Proc. 2009 3rd IEEE International Conf. on Digital Ecosystems and Technologies, pp. 733-738.

[6] A. Carcano, A. Coletta, M. Guglielmi, M. Masera, I. N. Fovino, and A. Trombetta, "A Multidimensional Critical State Analysis for Detecting Intrusions in SCADA Systems," IEEE Trans. Industrial Informatics, vol. 7, pp. 179-186, May. 2011.

[7] S. Cheung, B. Dutertre, M. Fong, U. Lindqvist, K. Skinner, and A. Valdes, "Using model-based intrusion detection for SCADA networks," In Proc. 2007 the SCADA Security Scientific Symposium, pp. 127-134.

[8] U. K. Premaratne, J. Samarabandu, T. S. Sidhu, R. Beresh, and T. JianCheng, "An Intrusion Detection System for IEC61850 Automated Substations," IEEE Trans. Power Delivery, vol. 25, pp. 2376-2383, Oct. 2010. [9] T. Morris, R. Vaughn, and Y. Dandass, "A Retrofit Network Intrusion Detection System for MODBUS RTU and ASCII Industrial Control Systems," in Proc. 2012 45th Hawaii International Conf. on System Science (HICSS), pp. 2338-2345.

[10] C. W. Ten, J. Hong, and C. C. Liu, "Anomaly Detection for Cybersecurity of the Substations," IEEE Trans. Smart Grid, vol. 7, pp. 865873, Dec. 2011.

[11] A. Valdes, S. Cheung, "Communication Pattern Anomaly Detection in Process Control Systems," in Proc. 2009 IEEE International Conf. on Technologies for Homeland Security, pp. 22-29.

[12] W. Gao, T. Morris, B. Reaves, and D. Richey, "On SCADA Control System Command and Response Injection and Intrusion Detection," in Proc. 2010 IEEE eCrime Researchers Summit, pp. 1-9.

[13] E. D. Knapp, Industrial Network Security: Securing Critical Infrastructure Networks for Smart Grid, SCADA, and Other Industrial Control Systems, New York: Elsevier, 2011, pp.60-61.

[14] J. Hurley, A. Munoz, and S. Sezer, "ITACA: Flexible, Scalable Network Analysis," in Proc. 2012 IEEE International Conf. on Communications Industry Forum \& Exhibit., pp.1084-1088.

[15] C. L. Abad and R. I. Bonilla, "An Analysis on the Schemes for Detecting and Preventing ARP Cache Poisoning Attacks," in Proc. 2007 27th International Conf. on Distributed Computing Systems Workshops, pp. 60-60.

[16] Z. Trabelsi and K. Shuaib, "Man in the Middle Intrusion Detection," in Proc. 2006 IEEE Global Telecommunications Conf., pp. 1-6.

[17] Y. Yang, K. McLaughlin, T. Littler, S. Sezer, E. G. Im, Z. Q. Yao, B. Pranggono, and H. F. Wang, "Man-in-the-Middle Attack Test-bed Investigating Cyber-security Vulnerabilities in Smart Grid SCADA Systems," in Proc 2012 IET International Conf. on Sustainable Power Generation and Supply (SUPERGEN), pp. 1-8.

[18] J. C. Foster, Metasploit Toolkit for Penetration Testing, Exploit Development, and Vulnerability Research. Syngress Publishing, 2007

[19] IEEE Standard Communication Delivery Time Performance Requirements for Electric Power Substation Automation, IEEE Standard 1646-2004, Feb. 2005.

[20] QRadar SIEM. [Online]. Available: http://q1labs.com/Products/QRadarSIEM.aspx

[21] E. Egozcue, D. H. Rodríguez, J. A. Ortiz, V. F. Villar, and L. Tarrafeta. (2012, Jul.). Smart Grid Security: Recommendations for Europe and Member States. ENISA, Heraklion, GR. [Online]. Available: http://www.enisa.europa.eu

[22] S. Edgar and A. Burns, "Statistical analysis of WCET for scheduling," in Proc. $200122^{\text {nd }}$ IEEE Real-Time Systems Symposium, pp. 215-224.

[23] I. N. Fovino, A. Carcano, T. De Lacheze Murel, A. Trombetta, and M. Masera, "Modbus/DNP3 State-Based Intrusion Detection System," in Proc. 2010 24th IEEE Int'l Conf. on Advanced Information Networking and Applications, pp. 729-736. 